\title{
Microwave-Assisted Efficient Synthesis of Dialkylated Ureas from Ureas
}

\author{
Feng $\mathrm{Li}^{\mathrm{a}}$ and Juan $\mathrm{Ma}^{\mathrm{b}}$ \\ School of Chemical Engineering, Nanjing University of Science and Technology, P.R.China \\ ananjingfengli@163.com, b674654555@qq.com
}

Keywords: Dialkylated Ureas; Alcohols; Transition Metal; Microwave Irradiation; Synthetic Method

\begin{abstract}
Microwave irradiation provided an effective tool for rapid, clean, and high-yielding transformations of organic reactions. Despite these advances, the $\mathrm{N}$-alkylation of amines with alcohols under microwave irradiation remains less unexplored. We demonstrated a general and efficient method for the synthesis of dialkylated ureas from ureas under microwave irradiation is described. Reactios of ureas with a series of alcohols, such as benzyl alcohol, benzylic alcohols bearing a electron-donating group or a electron-withdrawing group, afforded the desired dialkylated ureas with good yields at a short time. Experimental results exhibited obvious advantages of microwave irradiation.
\end{abstract}

\section{Introduction}

The $\mathrm{N}$-alkylation of amines is one of the most important $\mathrm{C}-\mathrm{N}$ bond-forming reactions in the filed of organic synthesis.[1] Traditionally, the $N$-alkylation of amines was performed with alkyl halides in the presence stoichiometric bases. Recently, much attention has been paid to the $\mathrm{N}$-alkylation of amines with alcohols as environmentally benign alkylating agents instead of alkyl halides for the preparation of $N$-alkylated amines based on "hydrogen autotransfer" process, using iridium, ruthenium, or other transition metal catalysts.[2-8] In this process, alcohols are first dehydrogenated to form aldehydes with the generation of metal hydride species, then condensation between the resulting aldehydes and ketones occurs to afford imines, which undergo transfer hydrogenation to give the final $\mathrm{N}$-alkylated amines. This method is apparently attractive due to the formation of water as the only side product (Scheme 1).

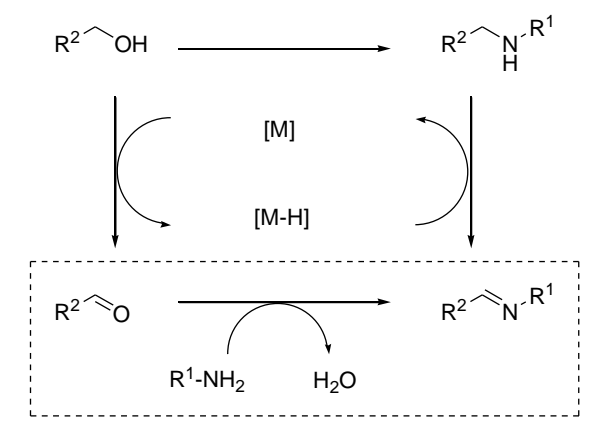

Scheme 1 Transition metal-catalyzed N-alkylation of amines with alcohols

In recent years, microwave irradiation has been widely employed as an effective reaction acceleration protocol, resulting in rapid, clean, and high-yielding transformations.[9] Despite these advances, the $\mathrm{N}$-alkylation of amines with alcohols under microwave irradiation remains less unexplored.[10] We have reported iridium-catalyzed synthesis of dialkylated ureas from ureas and alcohols.[11-12] The dialkylated ureas are important classess of biologically active compounds.However, the reaction required long reaction time (12 h).[13] Therefore, herein we wish to demonstrate that microwave irradiation significantly accelerated the process of the reaction. 


\section{Results and Discussion}

Our initial experiment focused on $\mathrm{N}$-alkylation of urea 1 with benzyl alcohol 2a. The reaction of was carried out in the presence of $\left[\mathrm{Cp}^{*} \mathrm{IrCl}_{2}\right]_{2}(0.4 \mathrm{~mol} \%) / \mathrm{KOH}\left(1\right.$ equiv.) at $110{ }^{\circ} \mathrm{C}$ under microwave irradiation. To our surprise, the dialkylated product 3aa could be obtained with $82 \%$ yield after reaction proceed for $2 \mathrm{~h}$ (Scheme 2$)$.

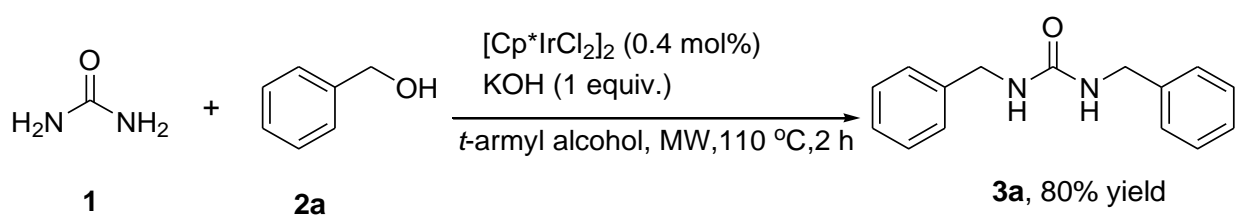

Scheme 2 N-alkylated of 1 with benzyl alcohol 2a under microwave irradiation

To explore the scope of reaction, the $N$-alkylation of $\mathbf{1}$ with various alcohols $\mathbf{2}$ was investigated under established conditions (Scheme 2) and these results were shown in Table 1. Reactions with benzylic alcohols bearing an electron-donating groups, such as 4-methyl $\mathbf{2 b}$, 4-isopropyl $\mathbf{2 c}$ and 4-methoxyl 2d, afforded the desired products 3b-3d with 68-72\% yields (Table 1, entries 1-3). Similarly, benzylic alcohols bearing one or two halogen atoms, such as 4-chloro 2e, 2,4-dichloro $2 \mathbf{f}$ and 4-bromo $\mathbf{2 g}$, were also proven to be suitable substrates, and the desired products $\mathbf{3 e - 3 g}$ were obtained with $77-80 \%$ yields (Table 1, entries 4-6). The $\mathrm{N}$-alkylation with benzylic alcohols bearing an electron-withdrawing trifluoromethyl $\mathbf{2 h}$ gave the corresponding products $\mathbf{3 h}$ with $73 \%$ yield (Table 1 , entry 7).

\section{Conclusions}

We have described a general and efficient method for the synthesis of dialkylated ureas from ureas under microwave irradiation. A series of products were obtained with high yields at a short time. Experimental results exhibited obvious advantages of microwave irradiation.

\section{Experimental Section}

General Experimental Details. Proton nuclear magnetic resonance $\left({ }^{1} \mathrm{H}\right.$ NMR) spectra were recorded at $500 \mathrm{MHz}$ using a Bruker Avance 500 spectrometer. Chemical shifts are reported in delta $(\delta)$ units, parts per million (ppm) downfield from trimethylsilane or ppm relative to the center of the singlet at $7.26 \mathrm{ppm}$ for $\mathrm{CDCl}_{3}$ and $2.50 \mathrm{ppm}$ for DMSO-d $\mathrm{d}_{6}$. Coupling constants $J$ values are reported in Hertz $(\mathrm{Hz})$, and the splitting patterns were designated as follows: s, singlet; $\mathrm{d}$, doublet; $\mathrm{t}$, triplet; $\mathrm{m}$, multiplet; b, broad. Carbon-13 nuclear magnetic resonance $\left({ }^{13} \mathrm{C} N \mathrm{NR}\right)$ spectra were recorded at $125 \mathrm{MHz}$ using a Bruker Avance 500 spectrometer. Chemical shifts are reported in delta $(\delta)$ units, ppm relative to the center of the triplet at $77.0 \mathrm{ppm}$ for $\mathrm{CDCl}_{3}$ and $39.5 \mathrm{ppm}$ for DMSO- $\mathrm{d}_{6} \cdot{ }^{13} \mathrm{C}$ NMR spectra were routinely run with broadband decoupling.

Microwave-Assisted synthesis of dialkylated ureas from ureas: Urea $(1 \mathrm{mmol})$, alcohol (2 $\mathrm{mmol}),\left[\mathrm{Cp}^{*} \mathrm{IrCl}_{2}\right]_{2}(1 \mathrm{~mol} \%), \mathrm{KOH}$ (1.0 mmol, 1.0 equiv.), $1 \mathrm{~mL}$ tert-amyl alcohol were added to a microwave vial containing a stirrer bar. The vial was then sealed before purging with nitrogen gas for 5 min. The mixture was then heated at $110{ }^{\circ} \mathrm{C}$ for $2 \mathrm{~h}$ under microwave irradiation $(300 \mathrm{~W}$, sealed reaction vessel), and was cooled to ambient temperature, concentrated in vacuo and purified by flash column chromatography with hexanes/ethyl acetate to afford the corresponding product.

1,3-dibenzylurea (3a). ${ }^{1} \mathrm{H}$ NMR $\left(500 \mathrm{MHz}, \mathrm{CDCl}_{3}\right) \delta$ 7.30-7.28 (m, $\left.4 \mathrm{H}, \mathrm{ArH}\right), 7.26-7.23(\mathrm{~m}, 6 \mathrm{H}$, $\mathrm{ArH}), 4.89(\mathrm{t}, J=5.0 \mathrm{~Hz}, 2 \mathrm{H}, \mathrm{NH}), 4.33\left(\mathrm{~d}, J=5.7 \mathrm{~Hz}, 4 \mathrm{H}, 2 \mathrm{xCH}_{2}\right) ;{ }^{13} \mathrm{C} \mathrm{NMR}\left(125 \mathrm{MHz}, \mathrm{CDCl}_{3}\right) \delta$ 
Table 2 N-Alkylation of $\mathbf{1 a}$ with various alcohols $\mathbf{2}$ under microwave irradiation $^{\text {[a] }}$

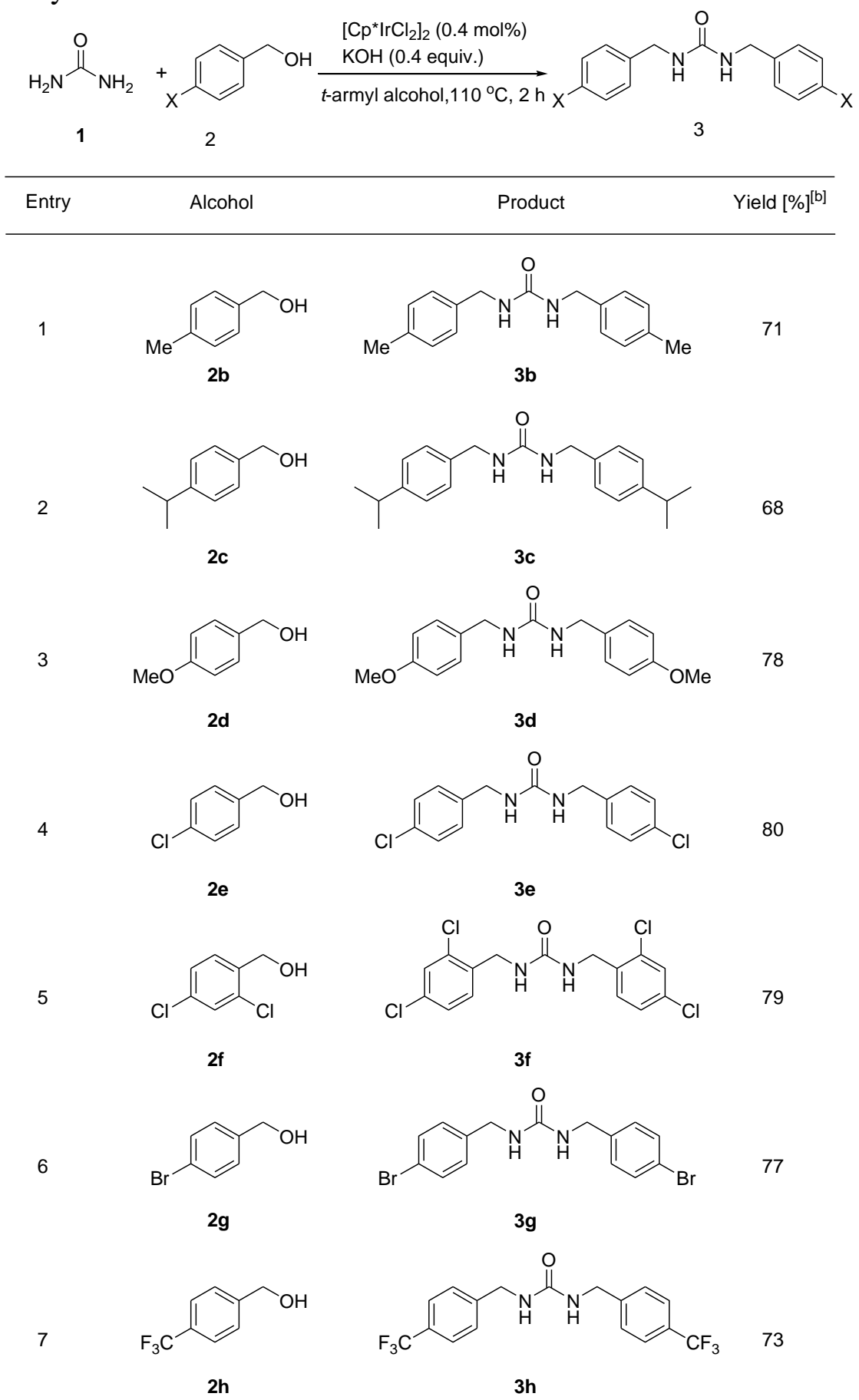

[a] Reaction conditions: $1 \mathrm{a}(1 \mathrm{mmol}), 2$ (2 mmol), $\left[\mathrm{Cp}^{*} \mathrm{IrCl}_{2}\right]_{2}(0.4 \mathrm{~mol} \%), \mathrm{KOH}(0.4$ equiv. $), t$-amyl alcohol $(1 \mathrm{~mL}), \mathrm{T}=110^{\circ} \mathrm{C}, \mathrm{t}=2 \mathrm{~h}$. [b] Isolatedyield.

$158.4,139.2,128.5,127.3,127.2,44.4$.

1,3-bis(4-methylbenzyl)urea (3b). ${ }^{1} \mathrm{H}$ NMR $\left(500 \mathrm{MHz}, \mathrm{DMSO}-\mathrm{d}_{6}\right) \delta 7.11$ (q, $J=6.95 \mathrm{~Hz}, 8 \mathrm{H}$, $\mathrm{ArH}), 6.42(\mathrm{t}, J=5.9 \mathrm{~Hz}, 2 \mathrm{H}, \mathrm{NH}), 4.16\left(\mathrm{~d}, J=5.9 \mathrm{~Hz}, 4 \mathrm{H}, \mathrm{CH}_{2}\right), 2.26\left(\mathrm{~s}, 6 \mathrm{H}, \mathrm{CH}_{3}\right) ;{ }^{13} \mathrm{C}$ NMR $(125$ MHz, DMSO-d $\left.{ }_{6}\right) \delta 158.43,138.21,135.63,129.28,127.63,43.01,21.15$. 
1,3-bis(4-isopropylbenzyl)urea (3c). ${ }^{1} \mathrm{H}$ NMR (500 MHz, DMSO-d 6 ) $\delta 7.16(\mathrm{~s}, 8 \mathrm{H}, \mathrm{ArH}), 6.33(\mathrm{t}, J$ $=5.2 \mathrm{~Hz}, 2 \mathrm{H}, \mathrm{NH}), 4.17\left(\mathrm{~d}, J=5.2 \mathrm{~Hz}, 4 \mathrm{H}, \mathrm{CH}_{2}\right), 2.85$ (quint, $\left.J=6.45 \mathrm{~Hz}, 2 \mathrm{H}, \mathrm{CH}\right), 1.18(\mathrm{~d}, J=6.6 \mathrm{~Hz}$, $\left.12 \mathrm{H}, \mathrm{CH}_{3}\right) ;{ }^{13} \mathrm{C}$ NMR $\left(125 \mathrm{MHz}, \mathrm{DMSO}-\mathrm{d}_{6}\right) \delta 158.29,146.96,138.48,127.35,126.33,43.04,33.36$, 24.21.

1,3-bis(4-methoxybenzyl)urea (3d). ${ }^{1} \mathrm{H}$ NMR (500 MHz, DMSO-d $\left.{ }_{6}\right) \delta 7.17$ (s, $\left.4 \mathrm{H}, \mathrm{ArH}\right), 6.87$ (s, $4 \mathrm{H}, \mathrm{ArH}), 6.29(\mathrm{~s}, 2 \mathrm{H}, \mathrm{NH}), 4.14\left(\mathrm{~s}, 4 \mathrm{H}, \mathrm{CH}_{2}\right), 3.72 \mathrm{ppm}\left(\mathrm{s}, 6 \mathrm{H}, \mathrm{OCH}_{3}\right) ;{ }^{13} \mathrm{C} \mathrm{NMR}(125 \mathrm{MHz}$, DMSO-d $\left.{ }_{6}\right) \delta 158.0,157.9,132.7,128.3,113.6,55.0,42.4$.

1,3-bis(4-chlorobenzyl)urea (3e). ${ }^{1} \mathrm{H}$ NMR $\left(500 \mathrm{MHz}, \mathrm{DMSO}-\mathrm{d}_{6}\right) \delta 7.36(\mathrm{~d}, J=8.00 \mathrm{~Hz}, 4 \mathrm{H}, \mathrm{ArH})$, $7.26(\mathrm{~d}, J=8.35 \mathrm{~Hz}, 4 \mathrm{H}, \mathrm{ArH}), 6.68(\mathrm{t}, J=5.95 \mathrm{~Hz}, 2 \mathrm{H}, \mathrm{NH}), 4.20\left(\mathrm{~d}, J=6.1 \mathrm{~Hz}, 4 \mathrm{H}, \mathrm{CH}_{2}\right) ;{ }^{13} \mathrm{C} \mathrm{NMR}$ (125 MHz, DMSO-d $\left.)_{6}\right) \delta 158.43,140.33,131.16,129.28,128.38,42.54$.

1,3-bis(2,4-dichlorobenzyl)urea (3f). ${ }^{1} \mathrm{H}$ NMR $\left(500 \mathrm{MHz}, \mathrm{DMSO}-\mathrm{d}_{6}\right) \delta 7.58(\mathrm{~s}, 2 \mathrm{H}, \mathrm{ArH}), 7.43(\mathrm{~d}$, $J=7.2 \mathrm{~Hz}, 2 \mathrm{H}, \mathrm{ArH}), 7.34(\mathrm{~d}, J=7.6 \mathrm{~Hz}, 2 \mathrm{H}, \mathrm{ArH}), 6.70(\mathrm{t}, J=5.6 \mathrm{~Hz}, 2 \mathrm{H}, \mathrm{NH}), 4.26(\mathrm{~d}, J=4.2 \mathrm{~Hz}$, $\left.4 \mathrm{H}, \mathrm{CH}_{2}\right) ;{ }^{13} \mathrm{C}$ NMR $\left(125 \mathrm{MHz}, \mathrm{DMSO}-\mathrm{d}_{6}\right) \delta 158.00,137.27,132.97,132.18,130.19,128.71,127.52$, 40.78 .

1,3-bis(4-bromobenzyl)urea (3g). ${ }^{1} \mathrm{H}$ NMR $\left(500 \mathrm{MHz}, \mathrm{DMSO}-\mathrm{d}_{6}\right) \delta 7.49(\mathrm{~d}, J=8.7 \mathrm{~Hz}, 4 \mathrm{H}, \mathrm{ArH})$, $7.20(\mathrm{~d}, J=8.7 \mathrm{~Hz}, 4 \mathrm{H}, \mathrm{ArH}), 6.66(\mathrm{t}, J=5.90 \mathrm{~Hz}, 2 \mathrm{H}, \mathrm{NH}), 4.18\left(\mathrm{~d}, J=6.25 \mathrm{~Hz}, 4 \mathrm{H}, \mathrm{CH}_{2}\right) ;{ }^{13} \mathrm{C} \mathrm{NMR}$ $\left(125 \mathrm{MHz}, \mathrm{DMSO}-\mathrm{d}_{6}\right) \delta 158.55,140.93,131.67,130.09,120.15,42.68$.

1,3-bis(4-(trifluoromethyl)benzyl]urea (3h). ${ }^{1} \mathrm{H}$ NMR (500 MHz, DMSO-d $\left.\mathrm{d}_{6}\right), 7.68(\mathrm{~d}, J=7.3 \mathrm{~Hz}$, $4 \mathrm{H}, \mathrm{ArH}), 7.46(\mathrm{~d}, J=7.2 \mathrm{~Hz}, 4 \mathrm{H}, \mathrm{ArH}), 6.68(\mathrm{t}, J=5.4 \mathrm{~Hz}, 2 \mathrm{H}, \mathrm{NH}), 4.31\left(\mathrm{~d}, J=4.8 \mathrm{~Hz}, 4 \mathrm{H}, \mathrm{CH}_{2}\right)$; ${ }^{13} \mathrm{C}$ NMR $\left(125 \mathrm{MHz}, \mathrm{DMSO}-\mathrm{d}_{6}\right) \delta 158.0,145.9,127.5,127.2\left(\mathrm{q}, J_{\mathrm{C}-\mathrm{F}}=31.6 \mathrm{~Hz}\right), 125.0\left(\mathrm{~d}, J_{\mathrm{C}-\mathrm{F}}=3.5\right.$ $\mathrm{Hz}), 124.3\left(\mathrm{q}, J_{\mathrm{C}-\mathrm{F}}=270.0 \mathrm{~Hz}\right), 42.6$.

\section{References}

[1] M. B. Smith and J. March, in March's Advanced Organic Chemistry, Wiley-Interscience, New York, 2001, pp. 499-501;

[2] M. H. S. A. Hamid, P. A. Slatford and J. M. J. Williams, Adv. Synth. Catal., 2007, 349, 1555;

[3] T. D. Nixon, M. K. Whittlesey and J. M. J. Williams, Dalton Trans., 2009, 753;

[4] G. E. Dobereiner and R. H. Grabtree, Chem. Rev., 2010, 110, 681;

[5] G. Guillena, D. Ramon and M. Yus, Chem. Rev., 2010, 110, 1611.

[6] K. Fujita, Z. Li, N. Ozeki, R. Yamaguchi, Tetrahedron Lett. 2003, 44, 2687

[7] K. Fujita, Y. Enoki, R. Yamaguchi, Tetrahedron 2008, 64, 1943.

[8] K. Fujita, A. Komatsubara, R. Yamaguchi, Tetrahedron 2009, 65, 3624.

[9] C. O. Kappe, Angew. Chem. Int. Ed. 2004, 43, 6250.

[10] I. Kim, H. Tsai, K. Nishi, T. Kasagami, C. Morisseau, B. D. Hammock, J. Med. Chem. 2007, 50, 5217.

[11] J. R. Falck, G. Wallukat, N. Puli, M. Goli, C. Arnold, A. Konkel, M. Rothe, R. Fischer, D. N. Muller, W. Schunck, J. Med. Chem. 2011, 54, 4109.

[12] W. Zhang, X. Dong and W. Zhao, Org. Lett. 2011, 13, 5386.

[13] F. Li, C. Sun, H. Shan, X. Zou and J. Xie, ChemCatChem, 2013, 5, 1543. 